\title{
On Positive-off-Diagonal Operators on Ordered Normed Spaces
}

\author{
A. Kalauch
}

\begin{abstract}
On a normed space $X$ ordered by a cone $K$ we consider a continuous linear operator $A: X \rightarrow X$ of the following kind: If a positive continuous functional $f$ attains 0 on some positive element $x$, then $f(A x) \geq 0$. If $X$ is a vector lattice, then such operators can be represented as $s I+B$, where $B$ is a positive operator, $I$ is the identity and $s \in \mathbb{R}$. We generalize this assertion for weaker assumptions on $X$, using the Riesz decomposition property.

Keywords: Positive-off-diagonal operators, ordered normed spaces, Riesz decomposition property
\end{abstract}

AMS subject classification: 47B60, 46B40, 47D03

\section{Introduction}

In the present paper let $(X, K,\|\cdot\|)$ be an ordered normed space, i.e. $X$ is a real vector space, $\|\cdot\|$ is a norm on $X$ and $K$ is a cone in $X$, i.e. $K$ is a wedge (i.e. $x, y \in K$ and $\lambda, \mu \geq 0$ imply $\lambda x+\mu y \in K)$ and $K \cap(-K)=\{0\}$. Furthermore, let $K$ be closed. By means of the cone $K$ a partial order is introduced in $X$. We will use the notations $x \in K$ and $x \geq 0$ synonymously and write $x>0$ instead of $0 \neq x \geq 0$. As usual, $X^{\prime}$ denotes the vector space of all continuous linear functionals on $X$ and $\mathcal{L}(X)$ the vector space of all continuous linear operators on $X$. An operator $B \in \mathcal{L}(X)$ is called positive if $B(K) \subseteq K$; a functional $f \in X^{\prime}$ is called positive if $f(K) \subseteq[0,+\infty)$. We write $B \geq 0$ and $f \geq 0$, correspondingly. The wedge of all positive functionals in $X^{\prime}$ is denoted by $K^{\prime}$. On $(X, K,\|\cdot\|)$ operators of the following kind are considered.

Definition 1.1. An operator $A \in \mathcal{L}(X)$ is called positive-off-diagonal if $x \in K$ and $f \in K^{\prime}$ with $f(x)=0$ imply $f(A x) \geq 0$.

Anke Kalauch: Inst. Anal. der TU, D-01062 Dresden, Germany

kalauch@math.tu-dresden.de

ISSN 0232-2064 / \$2.50 C Heldermann Verlag Berlin 
The notion "positive-off-diagonal" is motivated as follows: For $X=\mathbb{R}^{n}$ and the standard cone $K=\mathbb{R}_{+}^{n}$, a matrix $A=\left(a_{i j}\right)_{n, n}$ is a positive-offdiagonal operator if and only if $a_{i j} \geq 0$ for $i \neq j$. Note that on $\left(\mathbb{R}^{n}, \mathbb{R}_{+}^{n},\|\cdot\|\right)$ an invertible operator $A$, where the operator $-A$ is positive-off-diagonal and $A^{-1} \geq 0$, can be represented as a non-singular $M$-matrix and vice versa. ${ }^{1)}$ The set of all positive-off-diagonal operators on $(X, K,\|\cdot\|)$ is a wedge in $\mathcal{L}(X)$, but it is not a cone, since the identity $I$ and also $-I$ are both positiveoff-diagonal operators. If $A=s I+B$ with $B \geq 0$ and $s \in \mathbb{R}$, then the operator $A$ is positive-off-diagonal. If $X=\mathbb{R}^{n}$ and $K$ is a polyedral generating cone in $\mathbb{R}^{n}$, then the converse is also true, i.e. every positive-off-diagonal operator $A$ 2) can be represented as $A=s I+B$ where $s \in \mathbb{R}$ and $B \geq 0$ (see [6]). For several other cones in $\mathbb{R}^{n}$, in particular circular ones, this implication is not true (see [6] or Example 4.1 below).

On $(X, K,\|\cdot\|)$ consider for an operator $A \in \mathcal{L}(X)$ the properties

(i) $A$ is a positive-off-diagonal operator

(ii) $\|A\| I+A \geq 0$.

Obviously, (ii) implies (i). If $X$ is a Banach lattice, (i) and (ii) are equivalent (see, e.g., [4: C-II, Theorem 1.11]). We shall prove the implication (i) $\Rightarrow$ (ii) for operators on certain ordered normed spaces $X$ that need not be vector lattices. Note that for any $s \leq-\|A\|$ condition (ii) implies $B=-s I+A \geq 0$, hence $A=s I+B$ with $B \geq 0$.

There is a close connection between positive-off-diagonal operators and the theory of positive operator semigroups. Namely, consider the condition

(iii) $A$ is the generator of a semigroup $(T(t))_{t \geq 0}$ of positive operators on $X$.

Obviously, (ii) implies (iii) since

$$
T(t)=e^{t A}=e^{t(A+\|A\|)} e^{-t\|A\|} \geq 0 \quad(t \geq 0) .
$$

Now assume (iii) and consider $x \in K$ and

$$
A x=\lim _{t \downarrow 0} \frac{T(t) x-x}{t} .
$$

1) If $(X, K,\|\cdot\|)$ is an ordered normed space and $A \in \mathcal{L}(X)$ with $A=s I-B$, where $B \geq 0$ and $s>r(B)$ (here $r(B)$ denotes the spectral radius of the operator $B)$, then we call $A$ an $M$-operator. In the space $X=\mathbb{R}^{n}$ with the cone $K=\mathbb{R}_{+}^{n}$ the notion $M$-matrix is used.

2) Note that in Matrix Theory instead of "positive-off-diagonal" the notion "crosspositive" is used. 
Then for $f \in K^{\prime}$ with $f(x)=0$ one has

$$
f(A x)=\lim _{t \downarrow 0} \frac{f(T(t) x)-f(x)}{t}=\lim _{t \downarrow 0} \frac{f(T(t) x)}{t} \geq 0
$$

hence (iii) implies (i). In certain ordered normed spaces, e.g. if $K$ has a nonempty interior [3: Theorem 7.27], condition (i) implies (iii). In general (iii) does not imply (ii) (see Example 4.1). If one has the implication (i) $\Rightarrow$ (ii) for some ordered normed space, then this yields the equivalence of all properties (i), (ii) and (iii).

\section{Preliminaries}

Recall some definitions and notations of the theory of ordered vector spaces, where our terminology mainly follows that of $[1,7]$. Let $(X, K)$ be a real vector space ordered by a cone $K$. For given $a, b \in X$ with $a \leq b$, let $[a, b]=$ $\{x \in X: a \leq x \leq b\}$. $(X, K)$ is called Archimedean if $n x \in[0, y]$ for all $n \geq 1$ and some $y \in K$ implies $x=0$. An element $u>0$ is an order unit if for every $x \in X$ there exists a number $\lambda>0$ such that $x \in[-\lambda u, \lambda u] . K$ is generating if each $x \in X$ can be represented as $x=y-z$ where $y, z \in K$. The ordered vector space $(X, K)$ is said to satisfy the Riesz Decomposition Property, if for every $y, x_{1}, x_{2} \in K$ with $y \leq x_{1}+x_{2}$ there exist $y_{1}, y_{2} \in K$ such that $y=y_{1}+y_{2}$ and $y_{i} \leq x_{i} \quad(i=1,2)$.

An element $x>0$ is called an extremal of the cone $K$, if $y \in K$ and $y \leq x$ imply $y=\lambda x$ for some $\lambda \geq 0$, i.e. $x$ is an extremal of $K$ if and only if it generates an extreme ray of $K$. A subset $D$ of the cone $K$ is called a base of $K$ if $D$ is a non-empty convex set such that each $x>0$ has a unique representation $x=\lambda y$ with $y \in D$ and $\lambda>0$. If $K$ possesses a base $D$ with extreme points, then every extreme point of $D$ is an extremal of $K$.

Now let $(X, K)$ be a vector lattice. Note that every Dedekind complete vector lattice is Archimedean. A subset $S$ of $X$ is called solid, if $y \in X, x \in S$ and $|y| \leq|x|$ imply $y \in S$. The band generated by a singleton $\{x\}$, i.e. the intersection of all bands that contain the element $x$, will be denoted by $B_{x}$. Note that $B_{x}=\left\{y \in X:|y| \wedge n|x| \uparrow_{n}|y|\right\}$. Two vectors $x$ and $y$ are called disjoint, written $x \perp y$, if $|x| \wedge|y|=0$. The disjoint complement of a set $S \subset X$ is defined as $S^{d}=\{x \in X: x \perp y$ for all $y \in S\}$. A band $B$ in $X$ is called a projection band if $X=B \oplus B^{d}$.

Essentially we will make use of the following assertion (see [1: Theorem $3.8])$ :

Proposition 2.1. Every band in a Dedekind complete vector lattice is a projection band. 
Proposition 2.2. Let $(X, K)$ be an Archimedean vector lattice and $0<$ $x \in X$. The element $x$ is an extremal of $K$ if and only if $B_{x}=\{\lambda x: \lambda \in \mathbb{R}\}$.

Proof. Let $x$ be an extremal of $K$ and $y \in B_{x}$. Then $|y|=\sup \{|y| \wedge n|x|$ : $n \in \mathbb{N}\}$. Since $x$ is an extremal, $0 \leq|y| \wedge n|x| \leq n|x|=n x$ implies the existence of a number $\alpha_{n} \in \mathbb{R}$ such that $|y| \wedge n|x|=\alpha_{n} x$. We show that the sequence $\left(\alpha_{n}\right)_{n \in \mathbb{N}}$ is bounded. If the contrary is assumed, then for each $m \in \mathbb{N}$ there exists an $n \in \mathbb{N}$ such that $m x \leq \alpha_{n} x=|y| \wedge n|x| \leq|y|$. Since $X$ is Archimedean, we conclude $x=0$ which is a contradiction. If $C$ denotes an upper bound of $\left(\alpha_{n}\right)_{n \in \mathbb{N}}$, then

$$
|y|=\sup \{|y| \wedge n|x|: n \in \mathbb{N}\}=\sup \left\{\alpha_{n} x: n \in \mathbb{N}\right\} \leq C x .
$$

Since $x$ is an extremal, we get $|y|=\alpha x$ for some $\alpha \geq 0$. Finally, $y^{+}$and $y^{-}$are multiples of $x$ as well because of $0 \leq y^{+}, y^{-} \leq|y|=\alpha x$. Hence $y=y^{+}-y^{-}=\lambda x$ for some $\lambda \in \mathbb{R}$.

Vice versa, let $x \in K$ and $B_{x}=\{\lambda x: \lambda \in \mathbb{R}\}$. Obviously, since $B_{x}$ is solid, $0 \leq y \leq x$ implies $y \in B_{x}$, hence $y=\lambda x$

Now let $(X, K,\|\cdot\|)$ be an ordered normed space. A cone $K$ is called non-flat, if there exists a constant $\kappa>0$ such that each $x \in X$ possesses a representation $x=y-z$ with $y, z \in K$ and $\|y\|,\|z\| \leq \kappa\|x\|$. If $K$ has a nonempty interior, then $K$ is non-flat (and generating, obviously). $K$ is called normal, if the norm in $X$ is semi-monotone on $K$, i.e. there exists a constant (of semi-monotony) $N$ such that $0 \leq x \leq y$ implies $\|x\| \leq N\|y\|$. Note that $K^{\prime}$ is a cone in $X^{\prime}$ if and only if $X$ is the norm closure of $K-K$. We will call a non-empty subset $M \subseteq K^{\prime}$ total if $x \in X$ and $f(x) \geq 0$ for every $f \in M$ imply $x \in K$.

A norm $\|\cdot\|$ on a vector lattice is a lattice norm if $|x| \leq|y|$ implies $\|x\| \leq\|y\|$. If $(X, K,\|\cdot\|)$ is a normed vector lattice, i.e. a vector lattice equipped with a lattice norm, then for any two disjoint elements $x, y \in X$ one has

$$
\|x\| \leq\|x+y\| .
$$

This follows immediately from [1: Theorem 1.4] since

$$
0=|x| \wedge|y|=\frac{1}{2}(|x+y|-|x-y|)
$$

implies $|x-y|=|x+y|$ and

$$
|x| \leq|x| \vee|y|=\frac{1}{2}(|x+y|+|x-y|)=\frac{1}{2}(2|x+y|)=|x+y| .
$$

Since $\|\cdot\|$ is a lattice norm we get (1) 
Recall the following result of Riesz and Kantorovich [7: Theorem V.3.1]:

Proposition 2.3. If an ordered normed space $(X, K,\|\cdot\|)$ with a non-flat and normal cone $K$ satisfies the Riesz decomposition property, then $\left(X^{\prime}, K^{\prime}\right)$ is a Dedekind complete vector lattice.

In the case of a Banach space $X$ and a closed cone $K$, due to a theorem of Krein [7: Theorem III.2.1] the condition on $K$ to be non-flat can be replaced by the condition on $K$ to be generating. Note that because of $\left\|x^{+}\right\|,\left\|x^{-}\right\| \leq$ $\||x|\|=\|x\|$ any normed vector lattice satisfies all assumptions of Proposition 2.3 .

If $\left(X^{\prime}, K^{\prime}\right)$ is a Dedekind complete vector lattice, then for $f, g \in X^{\prime}$ and $x \in K$ one has

$$
(f \wedge g)(x)=\inf \left\{f\left(x^{\prime}\right)+g\left(x-x^{\prime}\right): x^{\prime} \in[0, x]\right\}
$$

and

$$
|f|(x)=\sup \{|f(y)|:|y| \leq x\} .
$$

In an ordered normed space $(X, K,\|\cdot\|)$ with a closed cone $K$ we consider for $0 \neq f \in K^{\prime}$ the following properties:

(I) $f^{-1}(0)=\left(f^{-1}(0) \cap K\right)-\left(f^{-1}(0) \cap K\right)$. This means that the part $f^{-1}(0) \cap K$ of the boundary of $K$ generates the corresponding hyperplane $f^{-1}(0)$ of $X$.

(II) $f$ is an extremal of $K^{\prime}$.

Property (I) always implies property (II). Indeed, for $g \in K^{\prime}$ with $0 \leq g \leq f$ from $f(x)=0$ for some $x \in X$ one has $x=x_{1}-x_{2}$ with $x_{1}, x_{2} \in f^{-1}(0) \cap K$, hence $0=f\left(x_{1}\right) \geq g\left(x_{1}\right) \geq g(x)$. Similarly, $f(-x)=0$ yields $0 \geq g(-x)$. This implies $g(x)=0$. Therefore we can conclude: Either $g=0$ or $g$ and $f$ have the same kernel. Hence $g=\lambda f$ for some $\lambda \in \mathbb{R}$.

In a Banach lattice property (II) also yields property (I). Indeed, for any $x \in X$ one has $x=x^{+}-x^{-}$, where $x^{+}=x \vee 0$. Suppose that $f$ is an extreme element in $K^{\prime}$ and $f(x)=0$. Then $f$ is a lattice homomorphism and we get $f\left(x^{+}\right)=f(0) \vee f(x)=0$. Accordingly, $f\left(x^{-}\right)=0$. However, in general property (II) does not imply property (I) (see Example 4.1).

We will say that a cone $K$ in an ordered normed space is b-generating if for every extremal $f$ of $K^{\prime}$ property (I) is satisfied. ${ }^{3)}$ Note that the Riesz decomposition property does generally not imply that $K$ is b-generating (consider, e.g., the space $X=C^{1}[0,1]$ of all continuously differentiable functions on $[0,1]$, ordered by the cone of non-negative functions).

3) This property is used, e.g., in [5]. 


\section{Main results}

We start with the main result on positive-off-diagonal operators.

Theorem 3.1. Let $(X, K,\|\cdot\|)$ be an ordered normed space that satisfies the Riesz decomposition property and let $K$ be a closed normal non-flat $b$ generating cone. Assume that there exists a total set of extremals of $K^{\prime}$. Then for any operator $A \in \mathcal{L}(X)$ the conditions

(i) $A$ is a positive-off-diagonal operator

(ii) $\|A\| I+A \geq 0$

are equivalent.

An ordered normed space that satisfies all assumptions of Theorem 3.1 need not be a vector lattice (see Example 4.2 below). For the proof of Theorem 3.1 we need some preliminary results.

Lemma 3.2. Let $(X, K,\|\cdot\|)$ be an ordered normed space that satisfies the Riesz decomposition property. Furthermore, let the cone $K$ be closed, normal and b-generating. Then for every extremal $f$ of $K^{\prime}$ there exists some constant $C>0$ such that the following property is satisfied: For every $y \in f^{-1}(1) \cap K$ there exists an element $z \in f^{-1}(1)$ such that $z \leq y$ and $\|z\| \leq C$.

Proof. Let $f$ be an extremal of $K^{\prime}$. Fix some element $y_{0} \in f^{-1}(1) \cap K$ and put $C=N\left\|y_{0}\right\|$, where $N$ is the constant of semi-monotony of the norm. Let $y \in f^{-1}(1) \cap K$. The element $x=y-y_{0}$ lies in $f^{-1}(0)$ and can be decomposed into $x=x_{1}-x_{2}$, where $x_{1}, x_{2} \geq 0$ and $x_{1}, x_{2} \in f^{-1}(0)$, since $K$ is b-generating. Hence we get $0 \leq y \leq y+x_{2}=x_{1}+y_{0}$. The Riesz decomposition property yields $y=w+z$, where $0 \leq w \leq x_{1}$ and $0 \leq z \leq y_{0}$. Due to $f\left(x_{1}\right)=0$ one has $f(w)=0$, hence $f(z)=1$. Moreover, $\|z\| \leq N\left\|y_{0}\right\|=C$

Theorem 3.3. Let $(X, K,\|\cdot\|)$ be an ordered normed space that satisfies the Riesz decomposition property. Furthermore, let the cone $K$ be closed, normal, non-flat and b-generating and let $f$ be an extremal of $K^{\prime}$. If $g \in X^{\prime}$ is such that $f \perp g$ and $g(x) \geq 0$ for each $x \in f^{-1}(0) \cap K$, then $g \in K^{\prime}$.

Proof. Proposition 2.3 ensures that $\left(X^{\prime}, K^{\prime}\right)$ is a vector lattice. Let $f$ be an extremal of $K^{\prime}$ and $g \in X^{\prime}$ such that $g \neq 0$ and $f \perp g$. For $x>0$ we get

$$
0=(f \wedge|g|)(x)=\inf \left\{f\left(x^{\prime}\right)+|g|\left(x-x^{\prime}\right): x^{\prime} \in[0, x]\right\}
$$

Hence for every $n \in \mathbb{N}$ there exists some $x_{n} \in[0, x]$ such that $f\left(x_{n}\right)+|g|(x-$ $\left.x_{n}\right) \leq \frac{1}{n}$. This implies $f\left(x_{n}\right) \leq \frac{1}{n}$ and also

$$
\left|g(x)-g\left(x_{n}\right)\right|=\left|g\left(x-x_{n}\right)\right| \leq|g|\left(x-x_{n}\right) \leq \frac{1}{n} .
$$


If $f\left(x_{n}\right)=0$, then the premise ensures $g\left(x_{n}\right) \geq 0$. If $f\left(x_{n}\right)>0$, we obtain a lower bound for $g\left(x_{n}\right)$ as follows: For the extremal $f$ of $K^{\prime}$ let $C$ be the constant from Lemma 3.2. Since $\frac{1}{f\left(x_{n}\right)} x_{n} \in K$ and $f\left(\frac{1}{f\left(x_{n}\right)} x_{n}\right)=1$, we get an element $z_{n} \in f^{-1}(1)$ such that $z_{n} \leq \frac{1}{f\left(x_{n}\right)} x_{n}$ and $\left\|z_{n}\right\| \leq C$. Then $w_{n}=$ $x_{n}-f\left(x_{n}\right) z_{n}$ lies in $f^{-1}(0) \cap K$ and one has

$$
\left\|x_{n}-w_{n}\right\|=f\left(x_{n}\right)\left\|z_{n}\right\| \leq f\left(x_{n}\right) C \leq \frac{C}{n} .
$$

The premise ensures $g\left(w_{n}\right) \geq 0$. Since

$$
\left|g\left(x_{n}\right)-g\left(w_{n}\right)\right| \leq\|g\|\left\|x_{n}-w_{n}\right\| \leq\|g\| \frac{C}{n}
$$

we conclude

$$
g\left(x_{n}\right) \geq-\frac{\|g\| C}{n} .
$$

Now we prove the assertion by way of contradiction. Suppose that there exists a vector $x>0$ such that $g(x)<0$. Put $n>\frac{\|g\| C+1}{-g(x)}$. Then $-g(x)>\frac{\|g\| C}{n}+\frac{1}{n}$. For the corresponding $x_{n}$ inequality (3) shows

$$
g\left(x_{n}\right)-g(x)>\frac{-\|g\| C}{n}+\frac{\|g\| C}{n}+\frac{1}{n}=\frac{1}{n}
$$

which contradicts (2)

Now we come to the

Proof of Theorem 3.1. We already mentioned that condition (ii) implies condition (i). Now assume that $A$ is a positive-off-diagonal operator. We have to show $\|A\| x+A x \in K$ for every $x \in K$. Since there exists a total set $M$ of extremals of $K^{\prime}$ it suffices to show $f(\|A\| x+A x) \geq 0$ for each $f \in M$. Fix some $f \in M$. Since $\left(X^{\prime}, K^{\prime}\right)$ is a Dedekind complete vector lattice, from Proposition 2.1 follows that $B_{f}$ is a projection band in $X^{\prime}$, i.e. $X^{\prime}=B_{f} \oplus B_{f}^{d}$. Since $\left(X^{\prime}, K^{\prime}\right)$ is Archimedean and $f$ is an extremal of $K^{\prime}$, Proposition 2.2 yields $B_{f}=\{\lambda f: \lambda \in \mathbb{R}\}$. This allows us to represent the element $A^{*} f$ as $A^{*} f=f_{1}+f_{2}$, where $f_{1}=\lambda f$ and $f_{2} \perp f$. If we show both

(a) $f_{2}$ is positive

(b) $|\lambda| \leq\|A\|$,

then we can conclude

$$
\begin{aligned}
f(\|A\| x+A x) & =\|A\| f(x)+\left(A^{*} f\right)(x) \\
& =\|A\| f(x)+\lambda f(x)+f_{2}(x) \\
& =(\|A\|+\lambda) f(x)+f_{2}(x) \\
& \geq 0 .
\end{aligned}
$$


Property (a): According to Theorem 3.3 it suffices to show $f_{2}(x) \geq 0$ for any $x \in K$ with $f(x)=0$. In this case $f_{1}(x)=0$ and $f(A x) \geq 0$ since $A$ is a positive-off-diagonal operator. Hence

$$
f_{2}(x)=\left(A^{*} f\right)(x)-f_{1}(x)=f(A x) \geq 0 .
$$

Consequently, $f_{2} \in K^{\prime}$.

Property (b): From inequality (1) we conclude

$$
|\lambda|\|f\|=\|\lambda f\| \leq\left\|\lambda f+f_{2}\right\|=\left\|A^{*} f\right\| \leq\|A\|\|f\|
$$

and hence $|\lambda| \leq\|A\|$

If $K$ is closed, then $K^{\prime}$ is total [7: Section II.4]. If, additionally, there exists an interior point $u$ of $K$, then $F_{u}=\left\{f \in K^{\prime}: f(u)=1\right\}$ is a $\sigma\left(X^{\prime}, X\right)$ compact base of $K^{\prime}$ [8: Theorem II.3.2] and the set of extreme points of $F_{u}$ is a total set of extremals of $K^{\prime}$. Hence the following conclusion is obvious.

Corollary 3.4. Let $(X, K,\|\cdot\|)$ be an ordered normed space that satisfies the Riesz decomposition property and let $K$ be a closed normal b-generating cone with non-empty interior. Then for any positive-off-diagonal operator $A \in \mathcal{L}(X)$ one has $\|A\| I+A \geq 0$.

\section{Examples}

First we present an example which shows that a positive-off-diagonal operator $A$ in general can not be represented as $A=s I+B$ with a positive operator $B$ and a number $s \in \mathbb{R}$, even if $A$ operates on a finite-dimensional space.

Example 4.1. We consider the ordered normed space $\left(\mathbb{R}^{3}, K,\|\cdot\|\right)$, where

$$
K=\left\{t\left(x_{1}, x_{2}, 1\right): x_{1}^{2}+x_{2}^{2} \leq 1 \text { and } t \geq 0\right\}
$$

is a circular cone (see Figure 1) and $\|\cdot\|$ is the Euclidean norm on $\mathbb{R}^{3}$. The cone $K$ is closed, normal and has a non-empty interior. $K$ is not b-generating and does not satisfy the Riesz decomposition property (see, e.g., [2]). Note that $K^{\prime}=K$. Consider the operator given by the matrix

$$
A=\left(\begin{array}{ccc}
-2 & 1 & 0 \\
-1 & -1 & 0 \\
0 & 0 & -1
\end{array}\right)
$$

Let $x \in K$ and $y \in K^{\prime}$ such that $\langle x, y\rangle=0$ and assume $x=\left(x_{1}, x_{2}, 1\right)$. Then $x_{1}^{2}+x_{2}^{2}=1$ and $y=\left(-x_{1},-x_{2}, 1\right)$. Hence $\langle A x, y\rangle=x_{1}^{2} \geq 0$, i.e. $A$ is a 
positive-off-diagonal operator with respect to $K$. Note that $A$ is the generator of a semigroup of positive operators. Moreover, there is no number $s$ such that $s I+A$ is positive. Indeed, for $v=(0,-1,1) \in K$ one has $(s I+A) v=$ $(-1,-s+1, s-1) \notin K$ for every $s \in \mathbb{R}$.

Figure 1: Illustration of Example 4.1

A similar example can be found, e.g., in [6]. Example 4.1 provides an operator with the additional property

$$
(-A)^{-1}=\frac{1}{3}\left(\begin{array}{ccc}
1 & 1 & 0 \\
-1 & 2 & 0 \\
0 & 0 & 3
\end{array}\right) \geq 0 .
$$

Indeed, if $y=\left(y_{1}, y_{2}, 1\right)$ with $y_{1}^{2}+y_{2}^{2} \leq 1$ and $x=(-A)^{-1} y=\left(x_{1}, x_{2}, x_{3}\right)$, then $x_{3}=1$ and

$$
\begin{aligned}
x_{1}^{2}+x_{2}^{2} & =\frac{1}{9}\left(y_{1}+y_{2}\right)^{2}+\frac{1}{9}\left(-y_{1}+2 y_{2}\right)^{2} \\
& =\frac{1}{9}\left(2 y_{1}^{2}+5 y_{2}^{2}-2 y_{1} y_{2}\right) \\
& \leq 1 \\
& =x_{3},
\end{aligned}
$$

hence $x \in K$. 
Referring to the remark in Section 1 on $M$-operators in the space $\left(\mathbb{R}^{n}, \mathbb{R}_{+}^{n}, \|\right.$ $\|)$, the operator $C=-A$ presents an example such that $-C$ is positive-offdiagonal, $C^{-1} \geq 0$ but $C$ can not be represented as an $M$-operator (see also Figure 1).

In the following example we consider an ordered normed space that is not a vector lattice, but satisfies all assumptions of Theorem 3.1.

Example 4.2. Let

$$
\begin{aligned}
& X=\{x \in C[0,4]: x(2)=x(1)+x(3)\} \\
& K=\{x \in X: x(t) \geq 0 \text { for all } t \in[0,4]\} .
\end{aligned}
$$

The ordered vector space $(X, K)$ satisfies the Riesz decomposition property, it is not a vector lattice, and with the maximum norm it becomes a Banach space where $K$ is closed (see [7: Section V.2]). Furthermore, the cone $K$ is normal. As an order unit we can choose the function $e$ with

$$
e(t)= \begin{cases}1 & \text { for } t \in[0,1] \cup[3,4] \\ t & \text { for } t \in[1,2] \\ -t+4 & \text { for } t \in[2,3]\end{cases}
$$

(note that $e \in \operatorname{int}(K)$ ). For any $x \in X$ one has $x=y-z$, where $y=\|x\| e$ and $z=\|x\| e-x$ are positive. Furthermore, $\|y\| \leq 2\|x\|$ and $\|z\| \leq 3\|x\|$, hence we get the constant of non-flatness $\kappa=3$.

A set of extremals of $K^{\prime}$ is the collection of the evaluation maps $\varepsilon_{t}$ (i.e. $\varepsilon_{t}(x)=x(t)$ for each $\left.x \in X\right)$ determined by the points $t \in[0,2) \cup(2,4]$. This set is total.

Finally, $K$ is b-generating. To see this fix $s \in[0,2) \cup(2,4]$ and $x \in$ $\varepsilon_{s}^{-1}(0)=\{x \in X: x(s)=0\}$. The element $x$ belongs to the vector lattice $C[0,4]$, where $x$ can be represented as $x=x^{+}-x^{-}$with the nonnegative functions $x^{+}(t)=\max \{0, x(t)\}$ and $x^{-}(t)=\max \{0,-x(t)\}$. Note that $x^{+}(s)=x^{-}(s)=0$. In order to show that $\varepsilon_{s}^{-1}(0) \cap K$ is generating in $\varepsilon_{s}^{-1}(0)$ consider the following two cases:

Case (a): If $x(1)$ and $x(3)$ have the same sign, say $x(1) \geq 0$ and $x(3) \geq 0$, then $x^{+}$and $x^{-}$belong to the subspace $X \subset C[0,4]$. Indeed, one has $x^{+}(1)=$ $x(1), x^{+}(3)=x(3)$ and $x(2)=x(1)+x(3) \geq 0$, hence $x^{+}(2)=x(2)=$ $x^{+}(1)+x^{+}(3)$ and therefore $x^{+} \in X$. Because of $x^{-}(1)=x^{-}(3)=x^{-}(2)=0$ one has $x^{-} \in X$. The case $x(1) \leq 0, x(3) \leq 0$ can be considered analogously.

Case (b): If $x(1)$ and $x(3)$ have different signs, say $x(1)>0$ and $x(3)<0$, then $x^{+}$and $x^{-}$may not belong to $X$. However, we can still find another representation $x=x_{1}-x_{2}$ such that $0 \geq x_{1}, x_{2} \in X$ with $x_{1}(s)=x_{2}(s)=0$. Let $0 \leq w \in C[0,4]$ with $w(1)=w(3)=w(s)=0$ and $w(2)=x(1)-x^{+}(2)$. 
Note that $w(2) \geq 0$. Indeed, if $x(2)<0$, then $x^{+}(2)=0 \leq x(1)$. If $x(2) \geq 0$, then $x^{+}(2)=x(2)=x(1)+x(3) \leq x(1)$. Put now $x_{1}=x^{+}+w$ and $x_{2}=x_{1}-x$. Then $x_{1} \geq 0$ and $x_{2}=x^{+}+w-\left(x^{+}-x^{-}\right)=w+x^{-} \geq 0$. Obviously, $x_{1}(s)=x_{2}(s)=0$. We show that $x_{1}, x_{2} \in X$. For $x_{1}$ this follows from

$$
\begin{aligned}
x_{1}(1)+x_{1}(3) & =x^{+}(1)+w(1)+x^{+}(3)+w(3) \\
& =x(1) \\
& =x(1)-x^{+}(2)+x^{+}(2) \\
& =w(2)+x^{+}(2) \\
& =x_{1}(2)
\end{aligned}
$$

i.e. $x_{1} \in X$. For $x_{2}$ we proceed as follows:

$$
\begin{aligned}
x_{2}(1)+x_{2}(3) & =x_{1}(1)-x(1)+x_{1}(3)-x(3) \\
& =x_{1}(1)+x_{1}(3)-(x(1)+x(3)) \\
& =x_{1}(2)-x(2) \\
& =x_{2}(2),
\end{aligned}
$$

therefore $x_{2} \in X$.

Note that the functional $\varepsilon_{2}$ is not an extremal of $K^{\prime}$, and in the subspace $\varepsilon_{2}^{-1}(0)$ the cone $\varepsilon_{2}^{-1}(0) \cap K$ is not generating. Consider for example $x \in \varepsilon_{2}^{-1}(0)$, where $x(1) \neq 0$, and assume $x=x_{1}-x_{2}$ for some $x_{1}, x_{2} \in \varepsilon_{2}^{-1}(0) \cap K$. Then $0=x_{1}(2)=x_{1}(1)+x_{1}(3) \geq 0$ implies, in particular, $x_{1}(1)=0$. Analogously, $x_{2}(1)=0$. Finally, $x(1)=x_{1}(1)-x_{2}(1)=0$ yields a contradiction.

The space $X$ in Example 4.2 satisfies all assumptions of Theorem 3.1 (and of Corollary 3.4, respectively), hence every positive-off-diagonal operator on $X$ is an operator of the kind $s I+B$ with positive $B$ and $s \in \mathbb{R}$.

\section{References}

[1] Aliprantis, C. D. and O. Burkinshaw: Positive Operators. London: Acad. Press 1985.

[2] Aliprantis, C. D., Tourky, R. and N. C. Yannelis: The Riesz-Kantorovich formula and general equilibrium theory. J. Math. Economics 34 (2000), 55 76.

[3] Clement, Ph. and H. J. A. M. Heijmans: One-Parameter Semigroups. Amsterdam et al.: North-Holland 1987.

[4] Nagel, R. J.: One-Parameter Semigroups of Positive Operators. Lect. Notes Math. 1184 (1986). 
[5] Schmidt, G. C.: Cones with faces, m-discrete ordered linear spaces and representations of discrete linear lattices. Lin. Alg. Appl. 78 (1986), 147 - 161.

[6] Schneider, H. and M. Vidyasagar: Cross-positive matrices. SIAM J. Num. Anal. 7 (1970), 508 - 519.

[7] Vulikh, B. Z.: Introduction to the Theory of Cones in Normed Spaces (in Russian). Kalinin (Russia): Kalinin Univ. 1977.

[8] Vulikh, B. Z.: Special Topics of the Geometry of Cones in Normed Spaces (in Russian). Kalinin (Russia): Kalinin Univ. 1978.

Received 18.02.2002; in revised form 06.12.2002 